\title{
Tiroidectomía endoscópica transoral por abordaje vestibular
}

\section{Transoral endoscopic thyroidectomy vestibular approach}

\author{
Francisca Fernández A. ${ }^{1,2}$
}

\section{Resumen}

En las últimas dos décadas, distintos tipos de abordajes extracervicales se han ido desarrollando en la cirugía endocrinológica, y principalmente en la tiroidectomía, con el fin de obtener mejores resultados cosméticos. Estos abordajes transfieren la incisión clásica descrita por Kocher, a puntos de acceso más discretos, sin embargo, requieren de disecciones extensas. Fue el desarrollo del abordaje vía vestibular el que cambió el paradigma terapéutico, ya que es el único potencialmente libre de cicatrices cutáneas, que ofrece la seguridad y resultados comparables con técnicas tradicionales. Además, la menor distancia entre el sitio de abordaje y la glándula tiroides, obvia la necesidad de extensas disecciones asociadas a otros tipos de abordajes remotos. La tiroidectomía endoscópica transoral por vía vestibular utiliza un instrumento laparoscópico convencional, vía vestíbulo oral, a través del espacio premandibular insuflado con $\mathrm{CO}_{2}$. Esta técnica se considera segura y reproducible. El presente artículo de revisión pretende describir la técnica quirúrgica, con la finalidad de aportar información que resulte relevante para la práctica clínica diaria.

Palabras clave: Tiroidectomía endoscópica, TOETVA, tiroidectomía transoral endoscópica.

\begin{abstract}
In the last two decades, different types of extra-cervical approaches have been described in endocrine surgery, mainly for thyroidectomy, to achieve better cosmetic outcomes. These approaches transfer the classic incision described by Kocher, to inconspicuous locations, yet they necessitate of extensive surgical dissections. It was the development of the vestibular approach that changed the treatment paradigm, as it is the only approach free of visible scars, with a safety profile and overall outcomes comparable to traditional techniques. Furthermore, the shorter distance between the access point and the thyroid gland, obviates the need for extensive dissections, such as those used in other remote approaches. The vestibular transoral endoscopic thyroidectomy uses a conventional laparoscopic instrument via the oral vestibule, through the premandibular space, which is insufflated with $\mathrm{CO}_{2}$. This technique is considered safe and reproducible. This review article aims to describe the surgical technique, in order to provide information relevant to routine clinical practice.
\end{abstract}

Keywords: Endoscopic thyroidectomy, TOETVA, transoral endoscopic thyroidectomy.

\section{${ }^{1}$ Servicio de}

Otorrinolaringología, Hospital

Carlos Van Buren. Valparaíso,

Chile.

${ }^{2}$ Departamento de

Especialidades, Cátedra de Otorrinolaringología, Escuela de Medicina, Facultad de Medicina, Universidad de Valparaíso. Viña del Mar, Chile.

La autora declara no tener conflictos de interés.

Recibido el 6 de agosto de 2020. Aceptado el 17 de enero de 2021.

Correspondencia: Francisca Fernández A. Dirección: cuatro $1 / 2$ poniente 472

Viña del Mar, Chile. Email: francisca.fernandez@ uv.cl

\section{Introducción}

En las últimas dos décadas, distintos tipos de abordajes se han ido desarrollando en la cirugía endocrinológica y principalmente en la tiroidectomía, con el fin de obtener resultados más cosméticos ${ }^{1}$. Considerando que la patología tiroidea afecta predominantemente a mujeres, los beneficios de una cirugía endoscópica podrían ser superiores a los de la cirugía convencional desde el punto de vista estético ${ }^{2}$. En 1999, Miccoli describe la técnica de tiroidectomía mínimamente invasiva video-asistida (MIVAT) realizada a través de una incisión única de $15 \mathrm{~mm}$ localizada en la región anterior del cuello, a dos centímetros 
de la escotadura esternal y sin insuflación ${ }^{3}$. Sin embargo, tres años antes ya se había descrito la primera paratiroidectomía usando insuflación con $\mathrm{CO}_{2}{ }^{4}$. Por otro lado, un número significativo de abordajes remotos en la glándula tiroides fueron desarrollados en forma endoscópica y vía robótica ; estos incluyen el acceso cervical lateral ${ }^{6}$, torácico ${ }^{7,8}$, axilar o combinado bilateral mamario-axilar ${ }^{9-11}$ y retroauricular ${ }^{12}$. Existen, además, técnicas con las cuales se consigue hacer disecciones en los niveles cervicales II, III y IV por abordaje endoscópico ${ }^{13}$. Si bien, todas estas técnicas han demostrado ser seguras, clínicamente efectivas y reproducibles en múltiples centros a nivel mundial, en todas persiste una cicatriz cutánea dependiente del sitio de abordaje ${ }^{14}$. Otra de las grandes críticas a estos abordajes extra-cervicales, son las extensas disecciones necesarias de realizar para acceder a la glándula tiroides, lo cual contradice la noción de una cirugía mínimamente invasiva. Por otro lado, la MIVAT no respetaría los planos anatómicos, contribuyendo a trastornos deglutorios y cicatrices visibles ${ }^{15}$. Se desarrolla entonces, como alternativa a este tipo de abordajes, la tiroidectomía transoral "Natural orifice trasluminal endoscopic surgery" (NOTES), inicialmente descrita por vía sublingual. Ésta evita cicatrices cutáneas visibles, y se caracteriza por una menor distancia entre el punto de acceso y la glándula tiroides, obviando las extensas disecciones necesarias en otras técnicas quirúrgicas ${ }^{15,16}$. Sin embargo, el acceso sublingual es abandonado por el significativo daño tisular asociado a la apertura del piso de boca. Se observan complicaciones tales como embolia por dióxido de carbono, enfisema mediastínico, infección de espacios cervicales profundos, alta tasa de conversión a cirugía abierta, y dificultades técnicas debido a la limitación en el movimiento ${ }^{1,17-19}$. Además, la subsecuente descripción de un abordaje transoral vestibular de menor complicación contribuye al abandono de la técnica. El abordaje por vía vestibular o "Transoral endoscopic thyroidectomy vestibular approach" (TOETVA), utiliza un instrumento laparoscópico convencional, vía vestíbulo oral, a través del espacio premandibular insuflado con $\mathrm{CO}_{2}$. Esta técnica se considera segura y más fácil de practicar en comparación a otras técnicas ${ }^{1}$.

\section{Técnica quirúrgica ${ }^{20}$}

Paciente en decúbito supino, con hombros alzados y cuello ligeramente extendido. Anestesia general con intubación nasotraqueal y administración de antibiótico endovenoso 30 minutos previo a la incisión de la piel. Desinfección habitual de la piel, en caso del vestíbulo se utiliza clorhexidina al 0,05\%. Se debe realizar la cirugía con 3 cirujanos, el primer cirujano justo encima de la cabeza y el asistente junto al operador de la cámara a cada lado. Se inicia el procedimiento mediante una incisión en un plano horizontal de $10 \mathrm{~mm}$ en el centro del vestíbulo oral, en la mitad del labio inferior o incluso en un plano más anterior. Con monopolar se diseca el músculo mentalis hasta la protuberancia mentoniana. Con una aguja de Veress (instrumento utilizado para establecer el neumoperitoneo en la práctica de la cirugía laparoscópica) se infiltran $30 \mathrm{ml}$ de solución salina con adrenalina al 1:500.000 en el área cervical anterior. Con una pinza Kelly se debe disecar a través de la incisión hasta la altura del cartílago tiroides, generando así el espacio de trabajo. La hidrodisección y la pinza Kelly permitirá separar el plano subplatismal de los músculos pretiroideos. Se completa el espacio de trabajo con un disector de punta roma desplazado en forma de abanico. Se procede a la inserción de trocar de lapararoscopía de $10 \mathrm{~mm}$ e insuflación de $\mathrm{CO}_{2}$ hasta $6 \mathrm{mmHg}$, con un flujo de $15 \mathrm{lt} / \mathrm{min}$. Dos incisiones verticales de $5 \mathrm{~mm}$ y paralelos a la incisión horizontal se realizan con bisturí. Esta incisión debe ser lateral a los incisivos, ya que una incisión más medial conlleva un alto riesgo de lesión del nervio mentoniano, lo que se manifiesta con parestesias alrededor del labio inferior y del mentón en el período posoperatorio. Dos trocares de $5 \mathrm{~mm}$ son introducidos por las incisiones laterales. Por el puerto central se introduce una óptica de $10 \mathrm{~mm} \mathrm{y} 30^{\circ}$ y por los puertos laterales el instrumental de trabajo, que incluye: electrocauterio monopolar L-hook, dispositivo ultrasónico, aspiración y pinzas grasper. Con cauterio monopolar L-hook y un dispositivo ultrasónico se completa el espacio de trabajo, estableciendo como límites: a) hacia superior el cartílago tiroides; b) hacia lateral los bordes libres del músculo esternocleidomastoideo y 
c) hacia inferior la escotadura esternal. Luego, se realiza apertura de la musculatura pretiroidea en línea media para exponer la cápsula tiroidea a nivel del istmo, tráquea y disección respecto del lóbulo a trabajar. Con una seda $2 / 0$ se retraen los músculos pretiroideos en forma transcutánea.

La disección del lóbulo tiroideo se inicia con el lóbulo piramidal, extendiéndose inferiormente para dividir el istmo cerca del lóbulo contralateral. En un plano avascular entre el polo superior y el cartílago tiroides (espacio de Joll's) se debe exponer el pedículo tiroideo superior. Éste se debe seccionar lo más cercano posible a la cápsula tiroidea, con el objetivo de preservar la glándula paratiroides superior. En algunos casos es posible visualizar la rama externa del nervio tiroideo superior insertándose en el músculo cricotiroideo. Una vez seccionado el pedículo, se debe continuar la disección del polo superior, traccionando con las pinzas grasper hacia el lado contralateral. Este movimiento de medialización permite exponer mejor el surco traqueoesofágico, y disecar el nervio laríngeo recurrente en todo su recorrido en dirección cefalo-caudal. La neuromonitorización del nervio laríngeo recurrente es posible bajo este abordaje, sin embargo, presenta sus limitaciones en cuanto al número de puertos presentes y área en la cual se puede utilizar $^{21}$. Nuevamente, el plano de disección debe ser lo más cercano a la cápsula tiroidea. El ligamento de Berry será cuidadosamente dividido. Una vez completada la lobectomía, remover la cámara e introducir una bolsa de extracción por el trocar de $10 \mathrm{~mm}$. Reposicionada la cámara, se introduce el lóbulo dentro de la bolsa. Es necesario el retiro de los tres trocares para retirar con mayor facilidad el espécimen por la incisión del vestíbulo con la ayuda de un fórceps. En caso de tumores $>4 \mathrm{~cm}$, será necesario fragmentar el lóbulo previamente en el interior de la bolsa, teniendo cuidado de no destruir el nódulo. Cierre de la musculatura pretiroidea con Vycril 3/0 y vestíbulo oral con Vycril 4/0. Colocar un drenaje cervical sólo en caso de tiroidectomía total; en ese caso se coloca otro trocar de 5 $\mathrm{mm}$ bajo visión directa en la fosa supraclavicular, y a través de él se aboca un drenaje aspirativo de tipo Jackson Pratt.

\section{Comparación con la técnica tradicional}

En una serie de Anuwong y cols. ${ }^{22}$, que compara 422 pacientes sometidos a TOETVA y 216 a cirugía convencional, sólo 3 pacientes debieron convertirse a técnica abierta, siendo incluidos en el grupo de cirugía convencional. Ambos grupos eran comparables, excepto en el tamaño del tumor, siendo en promedio de $3,8 \mathrm{~cm}$ y $4,7 \mathrm{~cm}$ para los grupos de TOETVA y tradicional respectivamente. La decisión de cuál abordaje utilizar fue basada en las preferencias del paciente, tamaño tumoral y naturaleza de la enfermedad. En el grupo TOETVA se observó un significativo aumento del tiempo operatorio, lo que es consistente con otras series. Por otro lado, no se encontraron diferencias significativas en relación con las pérdidas sanguíneas o incidencia de complicaciones, con una tasa de parálisis temporal del nervio laríngeo recurrente e hipoparatiroidismo de $5,9 \%$ y $10,9 \%$ respectivamente, similar a los observado en otros abordajes ${ }^{21,22}$. Ningún paciente sometido a TOETVA sufrió parálisis del nervio laríngeo recurrente o hipoparatiroidismo en forma permanente. Otras complicaciones observadas, propias de este tipo de abordaje, son la parálisis del nervio mentoniano (1,5\%-4,13\%) $)^{23,24}$, que disminuye al lateralizar las incisiones verticales ubicándolas lateral al canino ${ }^{20}$, equimosis, enfisema, hematoma, perforación o quemaduras de la piel, e infección de la herida operatoria ${ }^{19}$.

En cuanto a la curva de aprendizaje, Razavi y cols. ${ }^{25}$ lograron demostrar una curva de aprendizaje menor a la observada en otros accesos remotos. Mientras las técnicas asistidas por robot requieren entre 35 a 50 casos para lograr la competencia del cirujano ${ }^{26-28}$, la tiroidectomía endoscópica transoral por vía vestibular requiere sólo de $11 \operatorname{casos}^{25}$. Se presentan los criterios de inclusión y exclusión actualizados en la $1^{\circ}$ Conferencia Internacional Thyroides NOTES (Tabla 1).

\section{Conclusión}

La técnica TOETVA parece ser segura y reproducible. El instrumental a utilizar suele estar presente en cualquier servicio de cirugía y por lo tanto, no requiere equipamiento especial 


\section{Tabla 1. Criterios de inclusión y exclusión en TOETVA}

\section{Criterios de inclusión}

Glándula tiroides con un diámetro que no exceda los $10 \mathrm{~cm}$

Nódulo tiroideo benigno

Microcarcinoma papilar sin evidencia de metástasis

Neoplasia folicular

Enfermedad de Graves controlada

Bocios subesternales grado 1

\section{Criterios de exclusión}

No apto para cirugía

Incapaz de tolerar anestesia

para su realización. Es necesario seleccionar los casos en forma adecuada y considerar la curva de aprendizaje para lograr obtener resultados comparables a los obtenidos en un abordaje tradicional. Si bien la TOETVA no reemplaza el abordaje abierto, es una alternativa para aquellos pacientes que deseen resultados más favorables desde el punto de vista cosmético.

\section{Bibliografía}

1. Anuwong A. Transoral endoscopic thyroidectomy vestibular approach: a series of the first 60 human cases. World J Surg. 2016;40:491-7.

2. Shimizu K, Tanaka S. Asian perspective on endoscopic thyroidectomy - a review of 193 cases. Asian J Surg. 2003;26:92-100. doi: 10.1016/S10159584(09)60228-1.

3. Miccoli P, Berti P, Coute M, Bendinelli C, Marcocci C. Minimally invasive surgery for thyroid small nodules: preliminary report. J Endocrinol Invest. 1999;22:849-851.

4. Gagner M. Endoscopic subtotal parathyroidectomy in patients with primary hyperparathyroidism. Br J Surg. 1996;83:875.

5. Singer MC, Seybt MW, Terris DJ. Robotic facelift thyroidectomy: I. Preclinical simulation and morphometric assessment. Laryngoscope. 2011;121:1631-5. doi: 10.1002/lary.21831.

6. Sebag F, Palazzo FF, Harding J, Sierra M, Ippolito G, Henry JF. Endoscopic lateral approach thyroid lobectomy: safe evolution from endoscopic parathyoidectomy. World J Surg. 2006;30:802-805.

7. Shimizu K. Minimally invasive thyroid surgery. Best Pract Res Clin Endocrinol Metab. 2001;15:123-137.

8. Takami H, Ikeda Y. Total endoscopic thyroidectomy.
Asian J Surg. 2003;26:82-85.

9. Benhidjeb T, Anders S, Bärlehner E. Total videoendoscopic thyroidectomy via Axillo-BilateralBreast-Approach (ABBA). Langenbeck's Arch Surg. 2006;391:48-49.

10. Bärlehner E, Benhidjeb T. Cervical scarless endoscopic thyroidectomy: Axillo-Bilateral-Breast Approach (ABBA). Surg Endosc. 2008; 22:154-157.

11. Shimazu K, Shiba E, Tamaki Y, Takiguchi S, Taniguchi E, Ohashi S, Noguchi S. Endoscopic thyroid surgery through the axillo-bilateral-breast approach. Surg Laparosc Endosc Percutan Tech. 2003;13:196-201.

12. Singer MC, Seybt MW, Terris DJ. Robotic facelift thyroidectomy: I. Preclinical simulation and morphometric assessment. Laryngoscope. 2011;121:1631-5. doi: 10.1002/lary.21831.

13. Yan H, Wang Y, Wang, P, Xie Q, Zhao Q. "Scarless" (in the neck) endoscopic thyroidectomy (SET) with ipsilateral levels II, III, and IV dissection via breast approach for papillary thyroid carcinoma: a preliminary report. Surg Endosc. 2015;29(8):21582163.

14. Shen X, Miao ZM, Lu W, et al. Clinical experience with modified Miccoli's endoscopic thyroidectomy for treatment of thyroid carcinoma in 86 cases. Eur J Med Res. 2013;18(1):51. doi:10.1186/2047783X-18-51.

15. Benhidjeb T, Wilhelm T, Harlaar J, Kleinrensink GJ, Schneider TA, Stark M. Natural orifice surgery on thyroid gland: totally transoral video-assisted thyroidectomy (TOVAT): report of first experimental results of a new surgical method. Surg Endosc. 2009;23(5):1119-1120. doi:10.1007/s00464-009-03470.

16. Witzel K, Von Rahden BH, Kaminski C, Stein HJ. Transoral access for endoscopic thyroid resection. Surg Endosc. 2008;22: 1871-5.

17. Karakas E, Steinfeldt T, Gockel A, Westermann R, Kiefer A, Bartsch DK. Transoral thyroid and parathyroid surgery. Surg Endosc. 2010;24:1261-1267.

18. Wilhelm T, Metzig A.Endoscopic minimally invasive thyroidectomy (eMIT): a prospective proof-ofconcept study in humans. World J Surg. 2011;35:543551.

19. Woo SH. Endoscope-assisted transoral thyroidectomy using a frenotomy incision. J Laparoendosc Adv Surg Tech A. 2014;24:345-349.

20. Anuwong A, Sasanakietkul T, Jitpratoom P, Ketwong K, Kim HY, Dionigi G, Richmon JD. Transoral endoscopic thyroidectomy vestibular approach (TOETVA): indications, techniques and results. Surg Endosc. 2018;32(1):456-465.

21. Erol V, Dionigi G, Barczyński M, Zhang D, Makay Ö. Intraoperative neuromonitoring of the RLNs during TOETVA procedures. Gland Surg. 2020;9:S129-S135. doi: 10.21037/gs.2019.11.21. 
22. Anuwong A, Ketwong K, Jitpratoom P, Sasanakietkul T, Duh QY. Safety and Outcomes of the Transoral Endoscopic Thyroidectomy Vestibular Approach. JAMA Surg. 2018;153(1):21-27. doi: 10.1001/ jamasurg.2017.3366.

23. Chen S, Zhao M, Qiu J. Transoral vestibule approach for thyroid disease: a systematic review. Eur Arch Otorhinolaryngol. 2019;276:297-304.

24. Shan L, Liu J. A Systemic Review of Transoral Thyroidectomy. Surg Laparosc Endosc Percutan Tech. 2018;28:135-8.

25. Razavi CR, Vasiliou E, Tufano RP, Russell JO. Learning Curve for Transoral Endoscopic Thyroid Lobectomy. Otolaryngol
Head Neck Surg. 2018;159(4):625-629. doi: 10.1177/0194599818795881.

26. Kandil EH, Noureldine SI, Yao L, Slakey DP. Robotic transaxillary thyroidectomy: an examination of the first one hundred cases. J Am Coll Surg. 2012;214:558564.

27. Lee J, Yun JH, Nam KH, Soh EY, Chung WY. The learning curve for robotic thyroidectomy: a multicenter study. Ann Surg Oncol. 2011;18:226-232.

28. Lee J, Yun JH, Choi UJ, Kang SW, Jeong JJ, Chung WY. Robotic versus endoscopic thyroidectomy for thyroid cancers: a multi-institutional analysis of early postoperative outcomes and surgical learning curves. J Oncol. 2012;2012:734541. 